International Journal of Pure and Applied Mathematics

Volume 109 No. 4 2016, 813-826

ISSN: 1311-8080 (printed version); ISSN: 1314-3395 (on-line version)

url: http://www.ijpam.eu

doi: 10.12732/ijpam.v109i4.5

\title{
RANDOM COMMON FIXED POINT THEOREM FOR RANDOM WEAKLY SUBSEQUENTIALLY CONTINUOUS GENERALIZED CONTRACTIONS WITH APPLICATION
}

\author{
Rashwan A. Rashwan ${ }^{1}$, Hasanen A. Hammad ${ }^{2}$ \\ ${ }^{1}$ Department of Mathematics \\ Faculty of Science \\ Assuit University \\ Assuit, 71516, EGYPT \\ ${ }^{2}$ Department of Mathematics \\ Faculty of Science \\ Sohag University \\ Sohag, 82524, EGYPT
}

\begin{abstract}
In this paper, we prove random common fixed point theorem for two pairs of random self mappings under a generalized contractive condition using subsequential continuity with compatibility of type (E). An example is given to justify our theorem. Our results in randomness extend and improve the results of S. Beloul [4]. Finally, we give an application to discuss the existence of a solution of random Hammerstein integral equations.
\end{abstract}

AMS Subject Classification: 47H10, 24H25

Key Words: polish spaces, random operators, compatible of type (E), weakly subsequentially continuous

\section{Introduction}

The notion of commuting mappings is given by Jungck [13] to establish a common fixed point theorem. Two self mappings $F$ and $T$ of a metric space

Received: July 14, 2016

Revised: $\quad$ August 18, 2016

Published: October 6, 2016

${ }^{\S}$ Correspondence author
(C) 2016 Academic Publications, Ltd.

url: www.acadpubl.eu 
$(X, d)$ are commuting if $F T x=T F x$ for all $x \in X$. Later, weakly commuting mappings are defined by Sessa [19] as a generalization of commuting mappings. A pair of self mappings $F$ and $T$ are weakly commuting if $d(F T x, T F x) \leq d(F x, T x)$, for all $x \in X$. In $[14,15,20]$ the concepts of compatible, compatible of type $(A)$, compatible of type $(B)$, compatible of type $(C)$, compatible of type $(P)$ and weakly compatible mappings are presented as a generalization of weakly commuting as follows: Two self mappings $F$ and $T$ are:

(1) compatible if

$$
\lim _{n \rightarrow \infty} d\left(F T x_{n}, T F x_{n}\right)=0,
$$

(2) compatible of type $(A)$ if

$$
\lim _{n \rightarrow \infty} d\left(F T x_{n}, T^{2} x_{n}\right)=0 \text { and } \lim _{n \rightarrow \infty} d\left(T F x_{n}, F^{2} x_{n}\right)=0,
$$

(3) compatible of type $(B)$ if

$$
\begin{aligned}
\lim _{n \rightarrow \infty} d\left(F T x_{n}, T^{2} x_{n}\right) & \leq \frac{1}{2}\left[\lim _{n \rightarrow \infty} d\left(F T x_{n}, F t\right)+\lim _{n \rightarrow \infty} d\left(F t, F^{2} x_{n}\right)\right], \\
\lim _{n \rightarrow \infty} d\left(T F x_{n}, F^{2} x_{n}\right) & \leq \frac{1}{2}\left[\lim _{n \rightarrow \infty} d\left(T F x_{n}, T t\right)+\lim _{n \rightarrow \infty} d\left(T t, T^{2} x_{n}\right)\right]
\end{aligned}
$$

(4) compatible of type $(C)$ if

$\lim _{n \rightarrow \infty} d\left(F T x_{n}, T^{2} x_{n}\right) \leq \frac{1}{3}\left[\lim _{n \rightarrow \infty} d\left(F T x_{n}, F t\right)+\lim _{n \rightarrow \infty} d\left(F t, F^{2} x_{n}\right)+\lim _{n \rightarrow \infty} d\left(F t, T^{2} x_{n}\right)\right]$, $\lim _{n \rightarrow \infty} d\left(T F x_{n}, F^{2} x_{n}\right) \leq \frac{1}{3}\left[\lim _{n \rightarrow \infty} d\left(T F x_{n}, T t\right)+\lim _{n \rightarrow \infty} d\left(T t, T^{2} x_{n}\right)+\lim _{n \rightarrow \infty} d\left(T t, F^{2} x_{n}\right)\right]$,

(5) compatible of type $(P)$ if

$$
\lim _{n \rightarrow \infty} d\left(F^{2} x_{n}, T^{2} x_{n}\right)=0,
$$

(6) compatible of type $(E)$ if

$$
\lim _{n \rightarrow \infty} T^{2} x_{n}=\lim _{n \rightarrow \infty} T F x_{n}=F t \text { and } \lim _{n \rightarrow \infty} F^{2} x_{n}=\lim _{n \rightarrow \infty} F T x_{n}=T t,
$$

whenever $\left\{x_{n}\right\}$ is a sequence in $X$ such that $\lim _{n \rightarrow \infty} F x_{n}=\lim _{n \rightarrow \infty} T x_{n}=t$, for some $t \in X$,

(7) weakly compatible if $F x=T x, x \in X$ implies $F T x=T F x$.

One can note that, commuting $\Rightarrow$ weakly commuting $\Rightarrow$ compatible and compatibility of type $(\mathrm{A}) \Rightarrow$ compatibility of type $(\mathrm{C})$, however compatibility 
(compatibility of type (A), compatibility of type (B) and compatibility of type (C)) are equivalent under the continuity of $F$ and $T$.

In 2009, Bouhadjera and G. Thobie [6] introduced the concept of subsequential continuity as follows:

Definition 1. Two self maps $F$ and $T$ of a metric space $(X, d)$ is said to be subsequentially continuous if there exists a sequence $\left\{x_{n}\right\}$ such that $\lim _{n \rightarrow \infty} T x_{n}=$ $\lim _{n \rightarrow \infty} F x_{n}=t$ for some $t \in X, \lim _{n \rightarrow \infty} F T x_{n}=F t$ and $\lim _{n \rightarrow \infty} T F x_{n}=T t$.

Motivated by Definition 1, S. Beloul [4] give the following definitions

Definition 2. Let $F$ and $T$ be two self mappings of a metric space $(X, d)$, the pair $\{F, T\}$ is said to be weakly subsequentially continuous, if there exists a sequence $\left\{x_{n}\right\}$ such that $\lim _{n \rightarrow \infty} F T x_{n}=F t$ and $\lim _{n \rightarrow \infty} T F x_{n}=T t$, whenever $\lim _{n \rightarrow \infty} T x_{n}=\lim _{n \rightarrow \infty} F x_{n}=t$ for some $t \in X$.

Definition 3. A pair $\{F, T\}$ of mappings is said to be $T$-subsequentially continuous if there exists a sequence $\left\{x_{n}\right\}$ such that $\lim _{n \rightarrow \infty} T x_{n}=\lim _{n \rightarrow \infty} F x_{n}=t$ for some $t \in X, \lim _{n \rightarrow \infty} T F x_{n}=T t$.

Definition 4. The pair $\{F, T\}$ is said to be $F$-subsequentially continuous if there exists a sequence $\left\{x_{n}\right\}$ such that $\lim _{n \rightarrow \infty} T x_{n}=\lim _{n \rightarrow \infty} F x_{n}=t$ for some $t \in X, \lim _{n \rightarrow \infty} F T x_{n}=F t$.

Remark 1 If the pair $\{F, T\}$ is $F$-subsequentially continuous (or $T$ subsequentially continuous), then it is weakly subsequentially continuous.

In 1950, by the Prague school of probabilistic, random fixed point theory was initiated in the works of Hans [11] and spacek [21] as stochastic extension of classical fixed point theorem in separable Banach spaces, it plays an important role in the theory of random integral and random differential equations, also random fixed point theory can applied in various areas as optimization, approximation theory, variational inequalities and others. After paper of Bharucha-Ried [5], the field of random fixed point became most widely specially in functional analysis. A large number of researchers focussing on various aspects of random fixed point theory, some of these works see [3, 1, 12, 16-18].

\section{Preliminaries}

Let $(\Omega, \Sigma)$ denotes a measurable space consisting of a set $\Omega$ and sigma algebra $\Sigma$ of subsets of $\Omega, X$ stands for a separable Banach space and $\xi_{n}: \Omega \rightarrow X$ is a 
measurable sequence

Definition 5. ([7, 2, 8, 9])

1. A mapping $T: \Omega \longrightarrow X$ is said to be measurable ( $\Sigma$-measurable) if for any open subset $U$ of $X, T^{-1}(U)=\{\omega: T(\omega) \cap U \neq \phi\} \in \Sigma$.

2.A mapping $T: \Omega \times X \longrightarrow X$ is random operator, if for each fixed $x \in X$, the mapping $T(., x): \Omega \longrightarrow X$ is measurable.

3. A random operator $T: \Omega \times X \longrightarrow X$ is continuous if $T(x,):. \Omega \longrightarrow X$ is continuous, for each $x \in \Omega$.

4. A measurable mapping $\xi: \Omega \longrightarrow X$ is random fixed point of a random operator $T: \Omega \times X \longrightarrow X$ if $T(\omega, \xi(\omega))=\xi(\omega)$ for each $\omega \in \Omega$.

Motivated by definitions of S. Beloul [4] in deterministic, we state the following definitions in stochastic which are used in prove of our result:

Definition 6. Let $X$ be a Polish space (separable complete metric space) and $T, F: \Omega \times X \longrightarrow X$ then $\{F, T\}$ is said to be weakly random subsequentially continuous if there exists a sequence $\left\{x_{n}(\omega)\right\}$ such that

$$
\lim _{n \rightarrow \infty} T\left(\omega, x_{n}(\omega)\right)=\lim _{n \rightarrow \infty} F\left(\omega, x_{n}(\omega)\right)=x(\omega),
$$

for some $x \in X$ and $\omega \in \Omega$,

$$
\begin{aligned}
\lim _{n \rightarrow \infty} F\left(\omega, T\left(\omega, x_{n}(\omega)\right)\right) & \\
& =F(\omega, x) \text { and } \lim _{n \rightarrow \infty} T\left(\omega, F\left(\omega, x_{n}(\omega)\right)\right)=T(\omega, x) .
\end{aligned}
$$

Definition 7. Let $X$ be a Polish space (separable complete metric space) and $T, F: \Omega \times X \longrightarrow X$ then $\{F, T\}$ is said to be weakly random subsequentially continuous if there exists a sequence $\left\{x_{n}(\omega)\right\}$ such that $\lim _{n \rightarrow \infty} T\left(\omega, x_{n}(\omega)\right)=$ $\lim _{n \rightarrow \infty} F\left(\omega, x_{n}(\omega)\right)=x(\omega)$ for some $x \in X$ and $\omega \in \Omega$,

$$
\begin{aligned}
\lim _{n \rightarrow \infty} F\left(\omega, T\left(\omega, x_{n}(\omega)\right)\right) & \\
& =F(\omega, x) \text { and } \lim _{n \rightarrow \infty} T\left(\omega, F\left(\omega, x_{n}(\omega)\right)\right)=T(\omega, x) .
\end{aligned}
$$

Definition 8. A random self mappings $F$ and $T$ are said to be: $1-T$-random subsequentially continuous if

$$
\lim _{n \rightarrow \infty} T\left(\omega, F\left(\omega, x_{n}(\omega)\right)\right)=T(\omega, x) .
$$

2- $F$-random subsequentially continuous if 


$$
\lim _{n \rightarrow \infty} F\left(\omega, T\left(\omega, x_{n}(\omega)\right)\right)=F(\omega, x)
$$

3- Random compatible of type $(E)$ if

$$
\begin{aligned}
\lim _{n \rightarrow \infty} T\left(\omega, T\left(\omega, x_{n}(\omega)\right)\right) & =\lim _{n \rightarrow \infty} T\left(\omega, F\left(\omega, x_{n}(\omega)\right)\right)=F(\omega, x) \\
\text { and } \lim _{n \rightarrow \infty} F\left(\omega, F\left(\omega, x_{n}(\omega)\right)\right) & =\lim _{n \rightarrow \infty} F\left(\omega, T\left(\omega, x_{n}(\omega)\right)\right)=T(\omega, x) .
\end{aligned}
$$

for some $x \in X, \omega \in \Omega$ and there exists a measurable sequence $\left\{x_{n}\right\}$ from $\Omega$ to $C$ such that $\lim _{n \rightarrow \infty} T\left(\omega, x_{n}(\omega)\right)=\lim _{n \rightarrow \infty} F\left(\omega, x_{n}(\omega)\right)=x(\omega)$.

Remark 2. (i) If the pair $\{F, T\}$ is $F$ - random subsequentially continuous (or $T$-random subsequentially continuous), then it is weakly random subsequentially continuous.

(ii) If the pair $\{F, T\}$ is $F$-random subsequentially continuous (or $T$-random subsequentially continuous), then it is random subsequentially continuous this contradicts deterministic case in (Example 3. [4]).

Example 1. Let $X=[0, \infty)$ with the usual metric under

$$
d(x, y)=|x(\omega)-y(\omega)|
$$

Let $\Omega=[0,1]$ and let $\sum$ be the sigma measurable algebra subset of $[0,1]$. Define $T, F: \Omega \times X \rightarrow X$ be any random operators as follows:

$$
T(\omega, x)=\left(\frac{1-\omega^{2}+2 x}{3}\right) \text { and } F(\omega, x)=\left(\frac{1-\omega^{2}+3 x}{4}\right)
$$

for all $\omega \in \Omega, x \in X$, also define the sequence of measurable mapping $x_{n}(\omega)$ : $\Omega \rightarrow X$ as $x_{n}(\omega)=\left\{1+\left(\frac{1}{n}\right)-\omega^{2}\right\}$. It is clear that for all $\omega \in \Omega, x \in X$

$$
\lim _{n \rightarrow \infty} T\left(\omega, x_{n}\right)=\lim _{n \rightarrow \infty} F\left(\omega, x_{n}\right)=1-\omega^{2}=x(\omega) \text { for all } \omega \in \Omega .
$$

By the simple calculations, we have

$$
\begin{aligned}
\lim _{n \rightarrow \infty} T\left(\omega, F\left(\omega, x_{n}(\omega)\right)\right) & =\lim _{n \rightarrow \infty} T(\omega, x(\omega))=1-\omega^{2}, \\
\lim _{n \rightarrow \infty} F\left(\omega, T\left(\omega, x_{n}(\omega)\right)\right) & =\lim _{n \rightarrow \infty} F(\omega, x(\omega))=1-\omega^{2}, \\
\lim _{n \rightarrow \infty} T\left(\omega, T\left(\omega, x_{n}(\omega)\right)\right) & =\lim _{n \rightarrow \infty} T\left(\omega, F\left(\omega, x_{n}(\omega)\right)\right)=F(\omega, x)=1-\omega^{2} \\
\text { and } \lim _{n \rightarrow \infty} F\left(\omega, F\left(\omega, x_{n}(\omega)\right)\right) & =\lim _{n \rightarrow \infty} F\left(\omega, T\left(\omega, x_{n}(\omega)\right)\right)=T(\omega, x)=1-\omega^{2} .
\end{aligned}
$$


Therefore:

(i) the pair $\{F, T\}$ is weakly random subsequentially continuous,

(ii) A random mapping $T$ is $T$-random subsequentially continuous and $F$ is $F$-random subsequentially continuous,

(iii) a random mappings $F$ and $T$ are random compatible of type $(E)$.

Let a function $\phi:[0, \infty) \rightarrow[0, \infty)$ is said to be contractive modulus if $\phi(t)<t$ for all $t>0$ (see[17]) and non decreasing such that $\phi(0)=0$.

In this paper, we will establish a stochastic version of random common fixed point theorem for the pairs of random operators using concept of random subsequentially continuous with compatible of type (E), and an example to satisfy our result. Also we use this result to find the existence and uniqueness of a solution of random integral equations.

S. Beloul [4] studied the following general contractive type mappings and proved some fixed point theorems for this mappings under weakly subsequentially continuous and compatible of type $(\mathrm{E})$ :

Let $X$ be a metric space, $A, B, S, T: X \rightarrow X$ are four self mappings such that for all $x, y \in X$,

$$
\psi(d(S x, T y)) \leq \psi(M(x, y))-\phi(M(x, y))
$$

where

$$
M(x, y)=\max \left\{d(A x, B y), d(A x, S x), d(B y, T y), \frac{d(A x, T y)+d(B y, S x)}{2}\right\}
$$

and $\psi \in \Psi, \phi \in \Phi$ (such that $\Psi$ is the set of all nondecreasing continuous function, $\psi: R_{+} \rightarrow R_{+}$such $\psi(x)=0 \Leftrightarrow x=0$ and $\Phi$ be the set of all lower semi-continuous functions, $\left.\phi: R_{+} \rightarrow R_{+} \operatorname{such} \phi(x)=0 \Leftrightarrow x=0\right)$. If the two pairs $\{A, S\}$ and $\{B, T\}$ are weakly random subsequentially continuous and random compatible of type (E). Then $A, B, S$ and $T$ have a unique common random fixed point in $X$.

We now prove the random analogue of S. Beloul [4] with $\psi=2 \phi$.

\section{Main Result}

Now, we introduce our result in the following theorem

Theorem 9. Let $X$ be a Polish space and F, T, $P, Q: \Omega \times X \rightarrow X$ are four random mappings satisfy 


$$
\begin{aligned}
& d(F(\omega, x), Q(\omega, y)) \leq \\
& \quad \phi\left(\max \left\{\begin{array}{c}
d(P(\omega, x), Q(\omega, y)), d(P(\omega, x), F(\omega, x)), \\
d(Q(\omega, y), T(\omega, y)), \frac{d(P(\omega, x), T(\omega, y))+d(Q(\omega, y), F(\omega, x))}{2}
\end{array}\right\}\right),
\end{aligned}
$$

for all $x, y \in X$, and $\omega \in \Omega$, where $\phi:[0, \infty) \rightarrow[0, \infty)$ is contractive modulus and non decreasing such that $\phi(0)=0$. If the two pairs $\{T, Q\}$ and $\{F, P\}$ are weakly random subsequentially continuous and random compatible of type $(E)$. Then $F, T, P$ and $Q$ have a unique common random fixed point in $X$.

Proof. Since the pair $\{T, Q\}$ is weakly random subsequentially continuous, then it is $T$-random subsequentially continuous and there exists a sequence measurable mappings $\left\{y_{n}(\omega)\right\}$ in $X$ such that

$$
\lim _{n \rightarrow \infty} T\left(\omega, y_{n}(\omega)\right)=\lim _{n \rightarrow \infty} Q\left(\omega, y_{n}(\omega)\right)=y(\omega)
$$

and

$$
\lim _{n \rightarrow \infty} T\left(\omega, Q\left(\omega, y_{n}(\omega)\right)\right)=T(\omega, y(\omega)),
$$

for all $\omega \in \Omega$. Also $T$ and $Q$ are random compatible of type $(\mathrm{E})$, therefore

$$
\begin{gathered}
\lim _{n \rightarrow \infty} T\left(\omega, T\left(\omega, y_{n}(\omega)\right)\right)=\lim _{n \rightarrow \infty} T\left(\omega, Q\left(\omega, y_{n}(\omega)\right)\right)=Q(\omega, y(\omega)), \\
\lim _{n \rightarrow \infty} Q\left(\omega, Q\left(\omega, y_{n}(\omega)\right)\right)=\lim _{n \rightarrow \infty} Q\left(\omega, T\left(\omega, y_{n}(\omega)\right)\right)=T(\omega, y(\omega)) .
\end{gathered}
$$

From (3.3) and (3.4), we get

$$
Q(\omega, y(\omega))=T(\omega, y(\omega))
$$

Again, since $F$ and $P$ are weakly random subsequentially continuous, then there exists a sequence measurable mapping $x_{n}(\omega)$, such that

$$
\lim _{n \rightarrow \infty} F\left(\omega, x_{n}(\omega)\right)=\lim _{n \rightarrow \infty} P\left(\omega, x_{n}(\omega)\right)=x(\omega)
$$

and

$$
\lim _{n \rightarrow \infty} F\left(\omega, P\left(\omega, x_{n}(\omega)\right)\right)=F(\omega, x(\omega)),
$$

for all $\omega \in \Omega$, and $x(\omega): \Omega \rightarrow X$ be measurable mapping. Also $F$ and $P$ are random compatible of type $(\mathrm{E})$, therefore

$$
\begin{gathered}
\lim _{n \rightarrow \infty} F\left(\omega, F\left(\omega, x_{n}(\omega)\right)\right)=\lim _{n \rightarrow \infty} F\left(\omega, P\left(\omega, x_{n}(\omega)\right)\right)=P(\omega, x(\omega)), \\
\lim _{n \rightarrow \infty} P\left(\omega, P\left(\omega, x_{n}(\omega)\right)\right)=\lim _{n \rightarrow \infty} P\left(\omega, F\left(\omega, x_{n}(\omega)\right)\right)=F(\omega, x(\omega)) .
\end{gathered}
$$


Then (3.7) and (3.8), leads to

$$
F(\omega, x(\omega))=P(\omega, x(\omega))
$$

Now, we claim that $F(\omega, x(\omega))=T(\omega, y(\omega))$. If not, substituting from (3.5) and (3.9) in (3.1), we get

$$
\begin{aligned}
& d(F(\omega, x), T(\omega, y)) \leq \phi\left(\max \left\{\begin{array}{c}
d(F(\omega, x), T(\omega, y)), 0,0 \\
, \frac{d(F(\omega, x), T(\omega, y))+d(T(\omega, y), F(\omega, x))}{2}
\end{array}\right\}\right) \\
\leq & \phi(\max \{d(F(\omega, x), T(\omega, y)), 0,0, d(F(\omega, x), T(\omega, y))\}) \\
= & \phi(d(F(\omega, x), T(\omega, y)) .
\end{aligned}
$$

Since $\phi$ is a contractive modulus, then

$$
d(F(\omega, x), T(\omega, y)) \leq \phi(d(F(\omega, x), T(\omega, y))<(d(F(\omega, x), T(\omega, y)),
$$

which is a contradiction. So

$$
F(\omega, x(\omega))=P(\omega, x(\omega))=T(\omega, y(\omega))=Q(\omega, y(\omega)),
$$

again, we will show that $x(\omega)=F(\omega, x(\omega))$. If not, then from (3.1) and the inequality (3.10), we get $d\left(F\left(\omega, x_{n}(\omega)\right), Q(\omega, y)\right) \leq \phi\left(\max \left\{\begin{array}{c}d\left(P\left(\omega, x_{n}(\omega)\right), Q(\omega, y)\right), \\ d\left(P\left(\omega, x_{n}(\omega)\right), F\left(\omega, x_{n}(\omega)\right)\right), \\ d(Q(\omega, y), T(\omega, y)), \\ \frac{d\left(P\left(\omega, x_{n}(\omega)\right), T(\omega, y)\right)+d\left(Q(\omega, y), F\left(\omega, x_{\mathrm{n}}(\omega)\right)\right)}{2}\end{array}\right\}\right)$

Putting $n \rightarrow \infty$ in above inequality and using (3.2), we have

$$
\begin{aligned}
& d(x(\omega), F(\omega, x(\omega)) \\
& \leq \phi\left(\max \left\{d(x(\omega), F(\omega, x(\omega))), 0,0, \frac{d(x(\omega), F(\omega, x(\omega))+d(F(\omega, x(\omega)), x(\omega))}{2}\right\}\right) \\
& =\phi(d(x(\omega), F(\omega, x(\omega)))) .
\end{aligned}
$$

From definition $\phi$, we obtain that

$$
\begin{aligned}
d(x(\omega), F(\omega, x(\omega)) & \leq \phi(d(x(\omega), F(\omega, x(\omega)))<d(x(\omega), F(\omega, x(\omega)) \\
\text { i.e. } d(x(\omega), F(\omega, x(\omega))) & <d(x(\omega), F(\omega, x(\omega))),
\end{aligned}
$$

this is a contradiction again, therefore

$$
x(\omega)=F(\omega, x(\omega)=P(\omega, x(\omega)) .
$$


If $x(\omega) \neq y(\omega)$, then from $(3.1),(3.10)$ and (3.11), we get

$$
\begin{aligned}
& d\left(F\left(\omega, x_{n}(\omega)\right), Q\left(\omega, y_{n}(\omega)\right)\right) \leq \\
& \quad \phi\left(\max \left\{\begin{array}{l}
d\left(P\left(\omega, x_{n}(\omega)\right), Q\left(\omega, y_{n}(\omega)\right)\right), d\left(P\left(\omega, x_{n}(\omega)\right)\right. \\
\left., F\left(\omega, x_{n}(\omega)\right)\right), d\left(Q\left(\omega, y_{n}(\omega)\right), T\left(\omega, y_{n}(\omega)\right)\right) \\
, \frac{d\left(P\left(\omega, x_{\mathrm{n}}(\omega)\right), T\left(\omega, y_{\mathrm{n}}(\omega)\right)\right)+d\left(Q\left(\omega, y_{\mathrm{n}}(\omega)\right), F\left(\omega, x_{\mathrm{n}}(\omega)\right)\right)}{2}
\end{array}\right\}\right)
\end{aligned}
$$

Taking the limit as $n \rightarrow \infty$ in above inequality and using (3.2), we obtain

$$
\begin{aligned}
d(x(\omega), y(\omega)) & \leq \phi\left(\max \left\{d(x(\omega), y(\omega)), 0,0, \frac{d(x(\omega), y(\omega))+d(y(\omega), x(\omega))}{2}\right\}\right) \\
& \leq \phi(d(x(\omega), y(\omega))) .
\end{aligned}
$$

From definition $\phi$, we get $d(x(\omega), y(\omega))<d(x(\omega), y(\omega))$ which is a contradiction again. Therefore $x(\omega)=y(\omega)$ is a common random fixed point of the four random mappings $F, T, P$ and $Q$.

For the uniqueness, let $z(\omega) \neq x(\omega)$ be another common random fixed point, then from (3.1), we have

$$
\begin{aligned}
& d(x(\omega), z(\omega)) \\
\leq & \left.\phi\left(\max \{d(x(\omega), z(\omega))), 0,0, \frac{d(x(\omega), z(\omega))+d(z(\omega), x(\omega))}{2}\right\}\right) \\
= & \phi(d(x(\omega), z(\omega)))<d(x(\omega), z(\omega)),
\end{aligned}
$$

we observe that $d(x(\omega), z(\omega))<d(x(\omega), z(\omega))$ a contradiction due to the wrong assumption $z(\omega) \neq x(\omega)$, so $z(\omega)=x(\omega)$. Therefore the four random mappings $F, T, P$ and $Q$ have a unique common random fixed point.

Putting $\phi(t)=\frac{1}{2} t, T=F$ and $Q=P$ in Theorem 3.1, we obtain the following corollary

Corollary 10. Let $X$ be a Polish space and T, $Q: \Omega \times X \rightarrow X$ are random mappings satisfy

$$
\begin{aligned}
d(T(\omega, x), Q(\omega, y)) \leq \\
\frac{1}{2} \max \left\{\begin{array}{c}
d(Q(\omega, x), Q(\omega, y)), d(Q(\omega, x), T(\omega, x)), \\
d(Q(\omega, y), T(\omega, y)), \frac{d(Q(\omega, x), T(\omega, y))+d(Q(\omega, y), T(\omega, x))}{2}
\end{array}\right\},
\end{aligned}
$$

for all $x, y \in X$ and $\omega \in \Omega$, If the pair $\{T, Q\}$ is weakly random subsequentially continuous and random compatible of type $(E)$. Then $T$ and $Q$ have a unique common random fixed point in $X$. 
Remark 3 we can prove Theorem 1 with the following conditions:

(i) $\{T, Q\}$ is $T$-random subsequentially continuous and $T$-random compatible of type(E) and $\{F, P\}$ is $F$-random subsequentially continuous and $F$-random compatible of type(E) or,

(ii) $\{T, Q\}$ is $Q$-random subsequentially continuous and $Q$-random compatible of type(E) and $\{F, P\}$ is $P$-random subsequentially continuous and $P$-random compatible of type(E) or,

(iii) $\{T, Q\},\{F, P\}$ random subsequentially continuous and $T$ or $Q$-random compatible ( $F$ or $P$-random compatible respectively) of type (E).

Now, we give an example which support Theorem 9:

Example 2 Let $(\Omega, \Sigma)$ denotes a measurable space and $C=\{1,2,3,4,5\} \subset$ $X=R$ with the usual metric $d$. Consider $\Omega=\{1,2,3,4,5\}$ and let $\sum$ be the sigma algebra of Lebesgue's measurable subset of $\Omega$. Define $T, Q, F, P: \Omega \times C \rightarrow$ $C$ by

$$
\begin{aligned}
& T(\omega, x)=\left\{\begin{array}{l}
2 \text { if } x=1 \\
5 \text { otherwise }
\end{array} \text { and } Q(\omega, x)=\left\{\begin{array}{l}
4 \text { if } x=1 \\
5 \text { otherwise }
\end{array}\right.\right. \\
& F(\omega, x)=\left\{\begin{array}{l}
3 \text { if } x=1 \\
5 \text { otherwise }
\end{array} \text { and } P(\omega, x)=\left\{\begin{array}{l}
1 \text { if } x=1 \\
5 \text { otherwise }
\end{array} \text { for all } \omega \in \Omega .\right.\right.
\end{aligned}
$$

Let us take $x_{n}(\omega)=x(\omega)=2$ and $\phi(t)=\frac{1}{2} t$, we have

$\lim _{n \rightarrow \infty} T\left(\omega, x_{n}(\omega)\right)=\lim _{n \rightarrow \infty} Q\left(\omega, x_{n}(\omega)\right)=\lim _{n \rightarrow \infty} F\left(\omega, x_{n}(\omega)\right)=\lim _{n \rightarrow \infty} P\left(\omega, x_{n}(\omega)\right)=5$,

for all $\omega \in \Omega$. Also, we get

$$
\begin{aligned}
& \lim _{n \rightarrow \infty} Q\left(\omega, T\left(\omega, x_{n}(\omega)\right)\right)=\lim _{n \rightarrow \infty} Q(\omega, T(\omega, 2))=\lim _{n \rightarrow \infty} Q(\omega, 5)=5, \\
& \lim _{n \rightarrow \infty} P\left(\omega, F\left(\omega, x_{n}(\omega)\right)\right)=\lim _{n \rightarrow \infty} P(\omega, F(\omega, 2))=\lim _{n \rightarrow \infty} T(\omega, 5)=5 .
\end{aligned}
$$

Moreover

$$
\begin{aligned}
\lim _{n \rightarrow \infty} T\left(\omega, T\left(\omega, x_{n}(\omega)\right)\right) & =\lim _{n \rightarrow \infty} T(\omega, T(\omega, 2))=\lim _{n \rightarrow \infty} T(\omega, 5)=5 \\
& =\lim _{n \rightarrow \infty} Q(\omega, 5)=\lim _{n \rightarrow \infty} Q(\omega, T(\omega, 2)) \\
& =\lim _{n \rightarrow \infty} Q\left(\omega, T\left(\omega, x_{n}(\omega)\right)\right), \\
\lim _{n \rightarrow \infty} F\left(\omega, F\left(\omega, x_{n}(\omega)\right)\right) & =\lim _{n \rightarrow \infty} F(\omega, F(\omega, 2))=\lim _{n \rightarrow \infty} F(\omega, 5)=5 \\
& =\lim _{n \rightarrow \infty} P(\omega, 5)=\lim _{n \rightarrow \infty} P(\omega, F(\omega, 2)) \\
& =\lim _{n \rightarrow \infty} P\left(\omega, F\left(\omega, x_{n}(\omega)\right)\right) .
\end{aligned}
$$


Therefore, the two pairs $\{T, Q\}$ and $\{F, P\}$ are weakly random subsequentially continuous and random compatible of type $(\mathrm{E})$. To satisfy the contractive condition (3.12), we consider the following cases:

(i) If we take $x, y \in C-\{1\}$, then the inequality is satisfied,

(ii) By taking $x=y=1$ in $C$, we get

$$
\begin{aligned}
1 & =d(F(\omega, x), Q(\omega, y)) \\
& \leq \phi\left(\max \left\{\begin{array}{c}
d(P(\omega, x), Q(\omega, y)), d(P(\omega, x), F(\omega, x)) \\
, d(Q(\omega, y), T(\omega, y)) \\
, \frac{d(P(\omega, x), T(\omega, y))+d(Q(\omega, y), F(\omega, x))}{2}
\end{array}\right\}\right) \\
& =\phi \max \{3,2,2,1\}=\phi(3)=\frac{3}{2} . \text { i.e. } 1<\frac{3}{2} .
\end{aligned}
$$

(iii) If we take $x=1$ and $y \in C-\{1\}$, we get

$$
\begin{aligned}
2 & =d(F(\omega, x), Q(\omega, y)) \\
& \leq \phi\left(\max \left\{\begin{array}{c}
d(P(\omega, x), Q(\omega, y)), d(P(\omega, x), F(\omega, x)) \\
, d(Q(\omega, y), T(\omega, y)) \\
, \frac{d(P(\omega, x), T(\omega, y))+d(Q(\omega, y), F(\omega, x))}{2}
\end{array}\right\}\right) \\
& =\phi \max \{4,2,0,3\}=\phi(4)=2 .
\end{aligned}
$$

(iv) If we take $y=1$ and $x \in C-\{1\}$, we have

$$
\begin{aligned}
1 & =d(F(\omega, x), Q(\omega, y)) \\
& \leq \phi\left(\max \left\{\begin{array}{c}
d(P(\omega, x), Q(\omega, y)), d(P(\omega, x), F(\omega, x)) \\
, d(Q(\omega, y), T(\omega, y)) \\
, \frac{d(P(\omega, x), T(\omega, y))+d(Q(\omega, y), F(\omega, x))}{2}
\end{array}\right\}\right) \\
& \leq \phi \max \{1,0,2,2\}=\phi(2)=1 .
\end{aligned}
$$

From the four cases, the contractive (3.1) is satisfied.

Consequently all conditions of Theorem 9 are satisfied, and $x(\omega)=5$ is a unique common random fixed point of the four mappings $F, T, P$ and $Q$.

\section{Application to Integral Equation}

Using Theorem 9, we discuss the existence and uniqueness of a solution of random integral equations (see [22]) as the following:

$$
x(\omega, t)=\int_{-\infty}^{\infty} K_{i}(\omega, s, t)(1+\sqrt{x(\omega, s)}) d s \text { for } i=1,2 \text { and } t, s \in R .
$$


where

(i) $R=(-\infty, \infty)$ is a compact with metric $d$ on $R \times R$,

(ii) $\omega \in \Omega$ is supporting set of measurable space $(\Omega, R)$ for each $R \subset \sum$,

(iii) $x(\omega, t)$ is the unknown vector-valued random variables for each $t \in R$,

(iv) $K(\omega, s, t)$ is the stochastic kernel defined for $t, s \in R$,

(v) $x(\omega, s)$ is vector-valued function of $s \in R$.

Let $C(\Omega, R)$ is the space of all continuous functions from $(\Omega \times R)$ into $R$, and it is a Banach space with a metric $d(x, y)=\sup |x-y|$.

Suppose that for all $x, y \in C(\Omega, R)$ there exists a $\delta \in(0,1)$ such that

$$
\int_{-\infty}^{\infty}(1+\sqrt{x(\omega, s)})\left|K_{1}(\omega, s, t)-K_{2}(\omega, s, t)\right| \leq \delta \phi(|x-y|)
$$

where $\phi$ is a contractive modulus and nondecreasing on $R$.

Then there exists a unique random solution of the system (4.1).

Proof. Consider two random mappings $F, Q: C(\Omega, R) \rightarrow C(\Omega, R)$ by

$$
F(x(\omega, t))=\int_{-\infty}^{\infty} K_{1}(\omega, s, t)(1+\sqrt{x(\omega, s)}) d s
$$

and

$$
Q(x(\omega, t))=\int_{-\infty}^{\infty} K_{2}(\omega, s, t)(1+\sqrt{x(\omega, s)}) d s
$$

for all $\omega \in \Omega, t, s \in R$. The system (4.1) has a random solution iff $F$ and $Q$ have a common random fixed point in $C(\Omega, R)$. Since $K_{i}$ is continuous, then $F$ and $Q$ are continuous then $\{I, F\},\{I, Q\}$ (where $I$ the identity random mappings on $C(\Omega, R))$ are sub-sequentially continuous and compatible of type (E). Moreover we get

$$
\begin{aligned}
|F(x(\omega, t))-Q(y(\omega, t))| & =\int_{-\infty}^{\infty}(1+\sqrt{x(\omega, s)})\left|K_{1}(\omega, s, t)-K_{2}(\omega, s, t)\right| \\
& \leq \delta \phi(|x-y|) \\
& \leq \phi(|x-y|) \\
& \leq \phi\left(\max \left\{\begin{array}{c}
d(P(\omega, x), Q(\omega, y)), \\
d(P(\omega, x), F(\omega, x)), \\
d(Q(\omega, y), T(\omega, y)), \\
\frac{d(P(\omega, x), T(\omega, y))+d(Q(\omega, y), F(\omega, x))}{2}
\end{array}\right\}\right)
\end{aligned}
$$


Therefore all conditions of Theorem 3.1 are satisfied with $P=T=I$. Then $F$ and $Q$ have a unique common random fixed point, so the system (4.1) have a unique random solution.

\section{References}

[1] I. Beg, M. Abbas, Common random fixed point of compatible random operators, Hindawi Pub. Corp., 2006 (2006), 1-15.

[2] I. Beg, M. Abbas, Iterative procedures for solution of random equations in Banach spaces, J. Math. Anal. Appl., 315 (2006), 181-201., 369-396.

[3] I. Beg, N. Shahzad, Random fixed point theorems for nonexpansive and contractive-type random operators on Banach spaces, J. Appl. Math. Stoch. Anal., 7 (1994), 569-580.

[4] S. Beloul, Common fixed point theorems for weakly subsequentially continuous generalized contractions with applications, J. App. Math. E-notes, 15 (2015), 173-186.

[5] A. T. Bharucha-Reid, fixed point theorems in probabilistic analysis, Bull. Amer. Math. Soc., 82 (1976), 641-657.

[6] H. Bouhadjera, C. G. Thobie, Common fixed point theorems for pairs of subcompatible maps, J. App.Math. E-notes, arXiv0906.3159v1 [math.FA],(2009)

[7] B. S. Choudhury, Convergence of a random iteration scheme to a random fixed point, $J$. Appl. Math. Stochastic Anal., 8 (1995), 139-142.

[8] B. S. Choudhury, M. Ray, Convergence of an iteration leading to a soulation of a random operator equation, J. Appl. Math. Stoch. Anal., 12 (1999), 161-168.

[9] B. S. Choudhury, A. Upadhyay, An iteration leading to random solutions and fixed points of operators, Soochow J.Math., 25 (1999), 395-400.

[10] V. Gupta, A. Saini, R. Kumar, Common fixed points for weakly compatible Maps in 2-metric space, Int. J. Math. Archive, 3 (2012), 3670-3675.

[11] O. Hans, Random operator equations, Proc. 4th Berkeley Symp. Math. Stat. Prob. Vol. II, Part I niversity of California Press, Berkeley, (1961), 185-202.

[12] S. Itoh, Random fixed-point theorems with an application to random differential equations in Banach spaces, J. Math. Anal. Appl., 67 (1979), 261-273.

[13] G. Jungck, ommuting mappings and fixed points, Amer. Math. Monthly, 83 (1976), 261263.

[14] G. Jungck, Compatible mappings and common fixed points, Int. J. Math. Math. Sci., 9 (1986), 771-779.

[15] G. Jungck, B. E Rhoades, ixed point for set valued functions without continuity, JIndian J. Pure Appl. Math., 29 1998), 227-238.

[16] T. C. Lin, Random approximations and random fixed point theorems for non-self maps, Proc. Am. Math. Soc., 103 ((1988), 1129-1135.

[17] D. Oregan, Fixed points and random fixed points for weakly inward approximable maps, Proc. Ameri. Math. Soc., 126 (1998), 3045-3053.

[18] V. M. Sehgal, C. Waters, Some random fixed point theorems for condensing operators, Proc. Amer. Math. Soc., 90 (1984), 425-429. 
[19] S. Sessa, On a weak commutativity condition of mappings in fixed point considerations, Publ. Inst. Math. (Beograd) (N.S.), 46 (1982) 149-153.

[20] M. R. Singh, Y. M. Singh, Compatible mappings of type (E) and common fixed point theorems of Meir-Keeler type, Int. J. Math. Sci. Engg. Appl., 1 (2007), 299-315.

[21] A. Spacek, zufăllige Gleichungen, Czechoslovak, Math. J., 80 (1955) 462-466.

[22] J. Yin, Z. Liu, Random fixed point theorems of random comparable operators and an application, Dis. Dynam. in Nat. and Soc., 2015 (2015), 1-6. 